\title{
Un « frisson achérontique »
}

Stefan George, ésotérisme et occultisme

\section{Ute Oelmann}

Traducteur : Jean-Louis Elloy

\section{(2) OpenEdition}

\section{Journals}

Édition électronique

URL : https://journals.openedition.org/rbnu/3199

DOI : 10.4000/rbnu.3199

ISSN : 2679-6104

\section{Éditeur}

Bibliothèque nationale et universitaire de Strasbourg

\section{Édition imprimée}

Date de publication : 1 novembre 2011

Pagination : 42-53

ISSN : 2109-2761

\section{Référence électronique}

Ute Oelmann, « Un « frisson achérontique » », La Revue de la BNU [En ligne], 4 | 2011, mis en ligne le 01 novembre 2011, consulté le 09 août 2021. URL : http://journals.openedition.org/rbnu/3199 ; DOI https://doi.org/10.4000/rbnu.3199

\section{(c) (i) (2)(2)}

La Revue de la BNU est mise à disposition selon les termes de la Licence Creative Commons Attribution - Pas d'Utilisation Commerciale - Partage dans les Mêmes Conditions 4.0 International. 


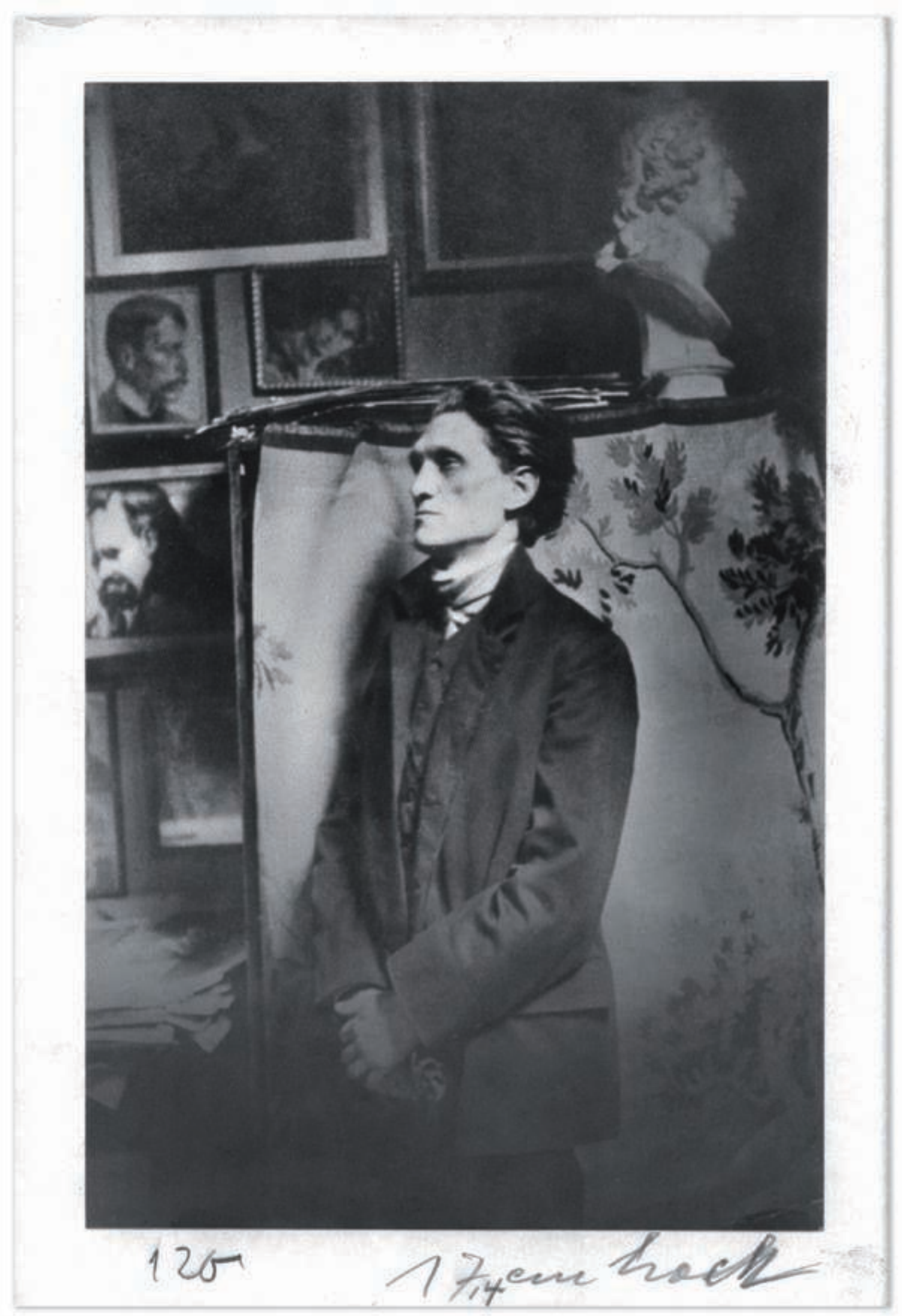

Stefan George dans l'atelier munichois du peintre et photographe Karl Bauer (coll. WLB) 


\section{UN «FRISSON ACHÉRONTIQUE » Stefan George, ésotérisme et occultisme}

\section{$\mathrm{U}$} n poète qui, en 1907, dédie un mémorial à un enfant défunt, et par le verbe et l'image fait de cet enfant un dieu, un poète qui crée une sorte d'évangile et confie à des artistes de l'illustration le soin d'orner de symboles, chrétiens comme antiques's son édition de luxe - édition que seuls peuvent connaître les initiés - un tel poète peut passer pour ésotérique, amateur d'occultisme, fondateur d'une religion, initiateur d'un culte. Un écrivain qui, dans ses poésies, célèbre " ce dieu de la jeunesse » avec un petit groupe d'amis, qu'il circonscrit à un cénacle choisi mais croissant, peut être soupçonné de sectarisme, d'élitisme, et au tournant du $20^{\mathrm{e}}$ siècle, il se retrouve en bonne compagnie. Cette époque a représenté un âge d'or pour l'occultisme et l'ésotérisme, et ce, d'une façon très sensible également, dans le domaine des arts. Ce phénomène a été largement décrit et étudié ces dernières décennies. Considéré au départ comme réactionnaire, en opposition à la modernité, le goût pour l'occultisme et pour l'ésotérisme a été ensuite compris comme intrinsèquement inscrit dans cette même modernité. La fascination pour l'occulte s'est associée aux préoccupations des sciences expérimentales, à la nouvelle science de la psychologie, au large intérêt manifesté à l'égard de l'anormal. De façon parallèle, la fascination pour les phénomènes de masse a crû avec celle pour les savoirs élitistes et mystérieux. Stefan George a pris part à toutes ces évolutions, à ces mouvements et contre-mouvements, mais - c'est ma thèse - tout en se tenant à distance de chacun d'eux.

En apparence on peut sans difficulté établir quels sont les courants dont George s'est réclamé, tant dans sa biographie que dans son œuvre poétique, mais ces choix s'accompagnent presque toujours de contradictions et de dénégations. On a bien du mal à ne pas quitter les sentiers battus de la littérature de témoignage et à ne pas reprendre cette perspective choisie dès le début pour appréhender et comprendre George. C'est ainsi que Robert Boehringer, ami, héritier du poète et son premier biographe ${ }^{2}$, a retenu le témoignage d'un compagnon d'école qui, plusieurs décennies plus tard, brosse le portrait de George comme celui d'un jeune homme conscient de son pouvoir, qui se targuait d'être doté de forces magiques qui lui permettaient de soumettre à ses volontés ses camarades du même âge. Il exigeait d'autrui la négation de la réalité, la perception devait céder la place à la foi (d'une piste de bowling il avait fait un sanctuaire); dans ce but, la pratique du mystère, du serment, le recours au cérémonial lui étaient d'un grand secours. Ce récit que les biographes et interprètes de George ont repris à loisir, Robert Boehringer l'a mis en parallèle, sans plus de commentaire, avec le début de l'activité poétique de George ; il suit la description suivante du jeune poète en dandy ironique : " Je t'imagine ainsi : assis en face de moi, dans ta chambre, le col de ton habit cramoisi relevé, ta main gauche jouant avec ton pince-nez sur la table, les pieds croisés... » ${ }^{3} \mathrm{Ce}$ dandy avait écrit des satires qu'il n’a pas conservées dans ses œuvres définitives mais Boehringer établit une corrélation entre le jeune magicien et l'authentique poète, lecteur d'Ibsen, de Pétrarque et du Tasse. A ce stade déjà, le jeune homme de dix-huit ans nous présente, tel Janus, un double visage, celui d'un mondain ironique et celui d'un magicien. Les poèmes que George a publiés en ces années sont l'œuvre d'un épigone ; c'est seulement par sa connaissance des Fleurs du mal, par ses lectures 
et traductions de Baudelaire auxquelles ont succédé celles de Mallarmé, de Verlaine et des jeunes symbolistes que George a trouvé, à partir de 1889 , son propre langage, qu'il a développé sa propre poétique. Certes tout cela est bien connu mais reste pourtant sous l'ombre d'un mystère, que George a lui-même désigné comme tel, lorsqu'il est revenu sur ses expériences parisiennes :

« Et dans la ville de la joie et de la grâce.../

Là, le héros et le poète, du Mystère montaient la garde :/

VILLIERS qui se sentait assez grand pour un trône/ VERLAINE, pieux et enfantin, dans la faute comme dans la pénitence,|

Et offrant son sang à son Idéal : MALLARMÉ $»^{4}$.

(Und in der heitren anmut stadt [...]

Da schirmten held und sänger das Geheimnis:

VILLIERS sich hoch genug für einen thron .

VERLAINE in fall und busse fromm und kindlich

Und für sein denkbild blutend: MALLARMÉ.)

Le poète allemand qui n'utilise habituellement que la minuscule orthographie " Mystère " avec une majuscule et ouvre ainsi un vaste champ d'interprétations possibles. Fait-il ici allusion aux enseignements à mystères d'Eliphas Lévi et de Joséphin Péladan, représentants des courants occultes, auteurs d'écrits occultes largement diffusés en France, qui ont sensiblement influencé les symbolistes français ? On en vint à interpréter ce passage non seulement comme le témoignage d'un hommage rendu à Mallarmé et aux symbolistes belges mais on y vit également la preuve certaine que George connaissait leurs sources occultes 5 . En vérité, nous n'avons conservé aucun témoignage comme quoi George se serait intéressé à Lévi ou à Péladan ou à leurs écrits de façon directe ou indirecte ; il ne les cite ni ne les mentionne ; mais quelle autre interprétation donner au terme de "Mystère " ? La prudence nous incite à rappeler que le poème dont il est question, Franken, a été écrit entre septembre 1898 et août 1900 et assurément par un poète qui " a déjà trouvé une assise, en lutte dans son sombre pays, encore incertain de la victoire ". Le " Mystère " n'est et ne fut autre que celui du chant, de la poésie, du Verbe. La première strophe de Franken parle de sa disparition en Allemagne, en ce $19^{\mathrm{e}}$ siècle finissant : "Où est ton poète, pauvre peuple plein d'orgueil ? Il n'en existe pas un seul ici ". Tel est le tableau que George dressait de l'Allemagne lorsqu'il la quitta pour l'Angleterre en 1888 puis pour la France, en quête d'une nouvelle écriture poétique.

Et pourtant l'on trouve, dans les imprimés laissés par George après sa mort, un exemplaire de la revue théosophique Sphinx. Mensuel pour la vie de l'âme et de l'esprit daté de janvier 1893. Éditée par Wilhelm Hübbe-Schleiden, la revue publiait des contributions à l'ambition scientifique sur des phénomènes occultes tels la télépathie ou le magnétisme. L’intérêt que George a manifesté à l'égard de ces thèmes dans les années suivantes doit plutôt être imputé à ses amis Melchior Lechter et Karl Wolfskehl - un véritable connaisseur de Péladan - et à l'influence des " Cosmiques " ("Kosmiker ") ${ }^{6}$ Alfred Schuler et Ludwig Klages, à Munich au tournant du siècle, et le numéro de 1893 ne semble l'avoir intéressé que dans la mesure où il se tournait vers la France. Il existe pourtant une autre explication. Le numéro laisse voir des traces de la lecture de George. Il s'est manifestement intéressé à des traductions de textes de Verlaine par Richard Dehmel (Wiedergeburt et $\mathrm{Zu}$ Gott) ${ }^{7}$ ainsi qu'à une lettre de ce dernier à son éditeur parue sous l'intitulé Zur Wiederverkörperung in der neuesten Literatur, qui se termine sur une auto-célébration et une auto-interprétation du poème Erlösungen que Dehmel venait de faire paraître ; George la commente de façon moqueuse en marge : « R. D. Immortel ». Son amie de Bingen, Ida Coblenz avait attiré peu de temps auparavant son attention sur ce poème, qui laissait apparaître " un fort tempérament ${ }^{8}$. La réaction de George prononça une condamnation sans appel : " un fort tempérament, on en trouve un aussi dans ce prétendu Lied der Menschheit (Chant de l'humanité)', ce qui n'empêche pas qu'il s'agisse d'une des créations les plus boursouflées et les plus dépourvues de talent qui soient ${ }^{10}$. Bien entendu, on ne peut exclure que George se soit intéressé à d'autres textes de la revue. Mais il n'existe pas d'autres traces concrètes de sa lecture.

Auparavant, en octobre 1892, était paru le premier numéro de la revue fondée par George, Blätter für die Kunst (Les feuillets pour l'art) et ce n'est que dans cette revue que George se livre à des considérations d'ordre esthétique ou poétique. Il ne les expose pas en son nom propre, il use du pronom " nous " qui représente la "Société des Feuillets pour l'art ", mais c'est une fiction littéraire au même titre que le travail d'édition de Carl August Klein, un ami de longue date de George. 


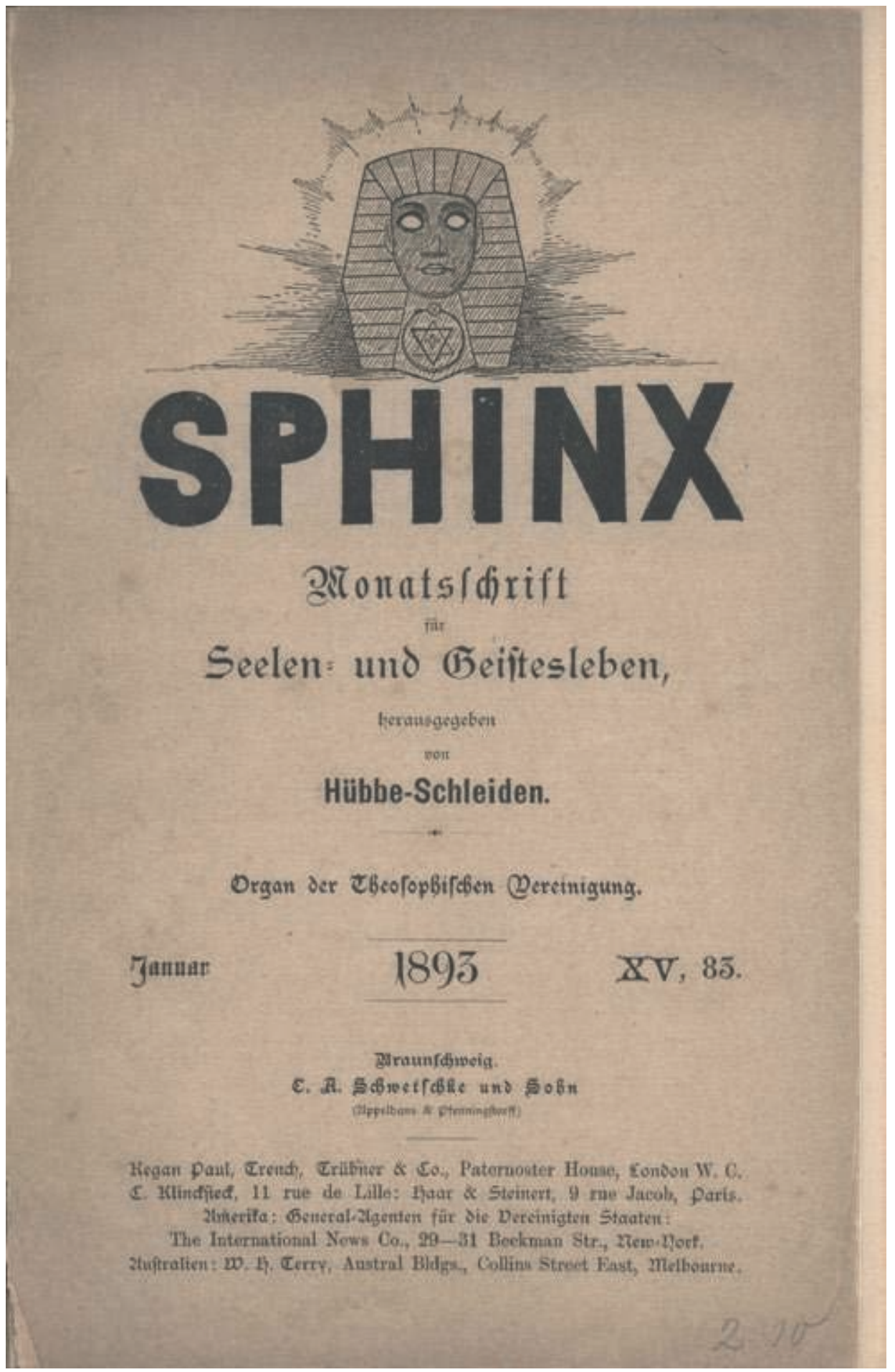

Couverture de la revue Sphinx de janvier 1893,

contenant des articles annotés par George (coll. WLB) 


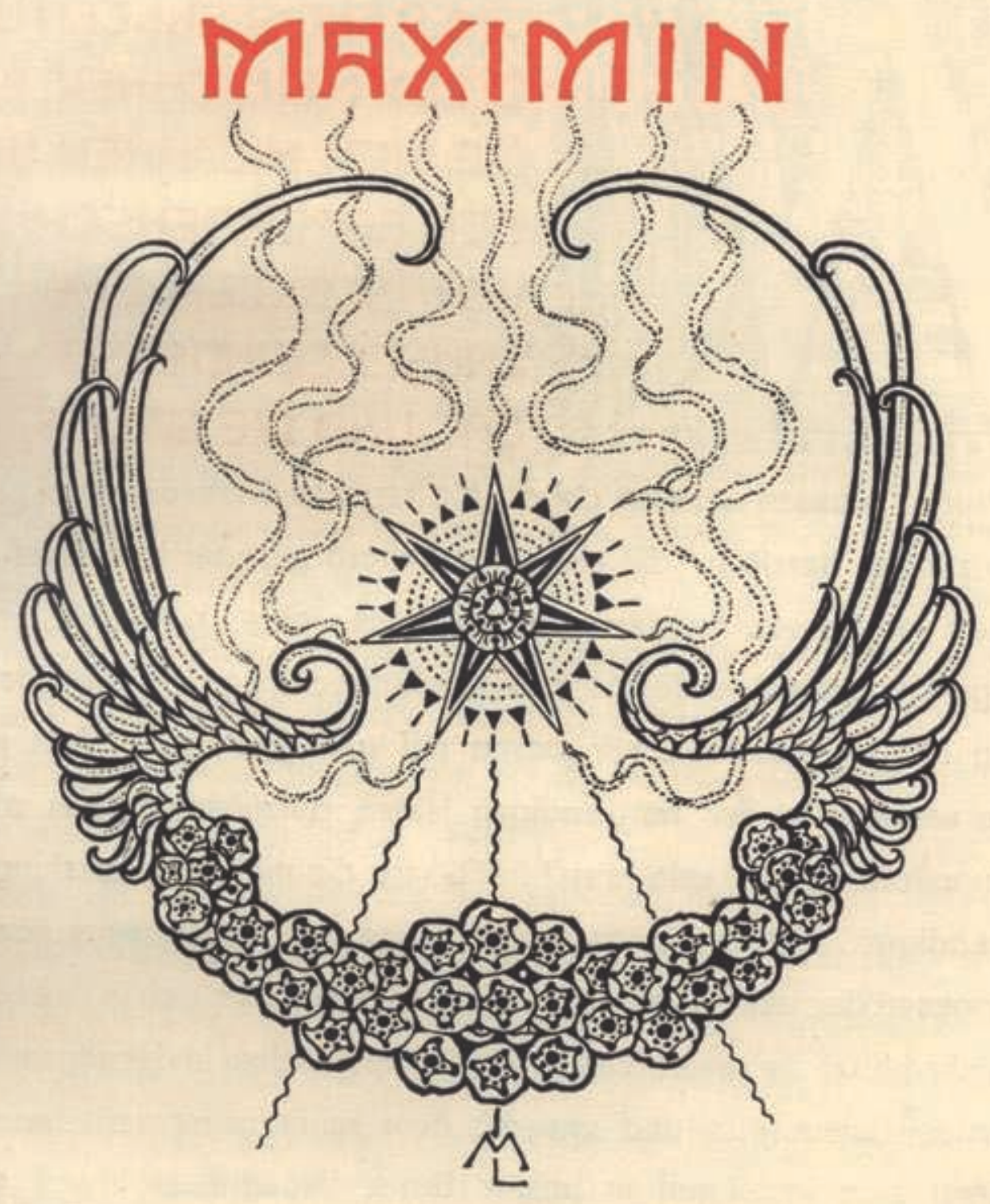

Illustration de Melchior Lechter dans l'ouvrage écrit par George en souvenir de Maximilian Kronberger

(1907 ; coll. WLB) 


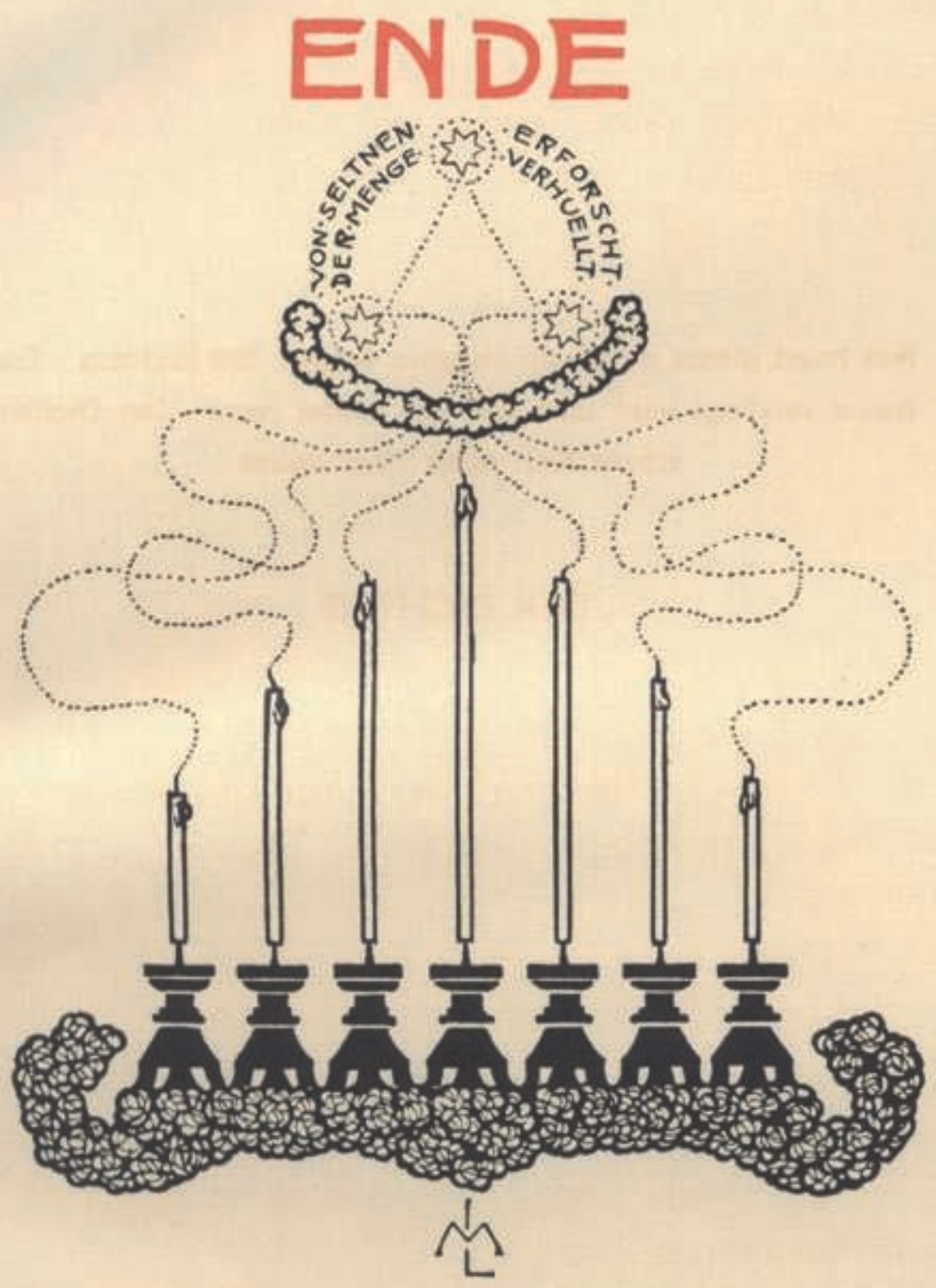

Illustration de Melchior Lechter dans l'ouvrage écrit par George en souvenir de Maximilian Kronberger

(1907 ; coll. WLB) 


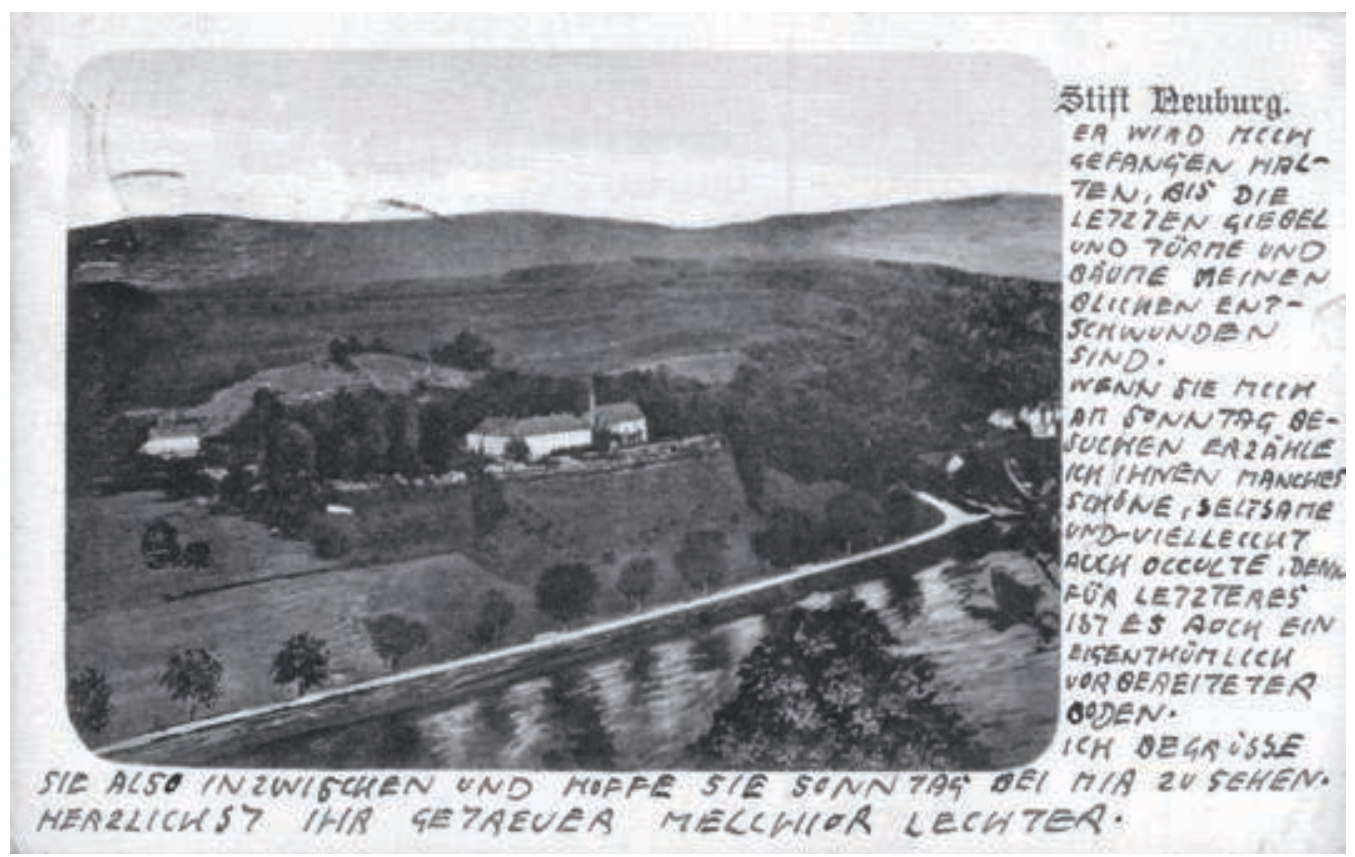

Melchior Lechter raconte les " nuits mystiques" au monastère de Neuburg (1909; coll. WLB)
La première suite de numéros annonce en toute clarté son credo : la publication veut " un art de nature spirituelle, sur la base d'une sensibilité nouvelle et s'engage à créer un art pour l'art ". Elle se tient à l'écart du réalisme et du naturalisme mais aussi de tout commerce avec " les projets visant à améliorer le monde ou avec les rêves de bonheur idéal ". A la fin de cette profession de foi est exprimée la croyance " en une éclatante renaissance dans l'art " et non dans la vie. C'est Dehmel qui croyait à une seconde naissance terrestre de l'homme, mais pas George. George a renouvelé avec quelques variations sa profession de foi dans le premier numéro de la troisième livraison de janvier 1896 : "Voici tout simplement ce que nous avons en partie désiré réaliser, ce que nous avons en partie perpétué : un art libre de toute finalité terrestre, qui se tient au-dessus de l'existence après l'avoir traversée, qui, à la façon de Zarathoustra, peut s'élever jusqu'à la plus haute mission de l'existence... un art porté par une joie visionnaire, fait d'ivresse, de son, de soleil ". Nous voilà bien loin de l'ésotérisme, des sciences occultes, de l'anormal, de l'obscur ! C'est à une haute exigence que sont astreints les artistes comme le public : " avec gravité et sainteté se rapprocher de l'art ", c'est ainsi que commence le deuxième numéro de la troisième livraison de la même année, et cela fait écho avec ce que la poétique et les œuvres de George vont confirmer avec toujours plus de force : leur étroit rapport avec une religion de l'art. Il n'est pas étonnant que dans le premier numéro de janvier 1896, la figure d'un ange entre en scène dans un poème de George, et annonce au poète un nouveau départ ${ }^{11}$. Ce n'est pas un ange de tradition chrétienne ; il est nu et annonciateur de " la vie belle ». En 1890 c'était encore une muse sous les traits d'une femme souveraine qui se tenait au seuil du premier poème de George, dans le premier recueil publié de ses poésies ${ }^{12}$; en 1896 , c'est une nouvelle vocation qui se fait jour, à laquelle George répondra en composant les vingt-quatre poésies du recueil Vorspiel (Prêlude), paru en 1900. Le langage religieux fait alors une entrée de plus en plus sensible dans l'œuvre poétique de George. A cela s'ajoute le travail d'illustrateur de Melchior Lechter, dont il ne faut pas sous-estimer l'influence; il atteint l'un de ses sommets dans le volume de 1900 Der Teppich des Lebens und die Lieder von Traum und Tod mit einem Vorspiel (Le tapis de la vie et les chants de rêve et de mort, précédés d'un prélude). Lechter renforce le syncrétisme de la langue religieuse de George en y ajoutant sa propre symbolique : ange, encens, ostensoir. Elle est encore fortement marquée par le catholicisme de Lechter mais s'enrichit ensuite de plus en plus, en fonction des centres d'intérêt nouveaux de ce dernier, et se mêle à des références à l'Inde, au bouddhisme et à la théosophie. Nous n'avons 


\section{"Ne confonds pas le prêtre avec le fakir, le 'vates' avec le mage, les esprits avec les fantômes ".}

aucune preuve d'une influence directe de George sur l'utilisation par Lechter de cette symbolique, quoique George, dans tous les autres domaines de l'édition, ait voulu avoir son mot à dire. Nous ne savons pas dans quelle mesure George a partagé l'intérêt de Lechter pour la théosophie. En 1903 toutefois, il a laissé dans l'appartement berlinois de Lechter trois numéros de la revue Die Gnosis. Mit den wissenschaftlichen Mitteilungen für Okkultismus (La gnose. Accompagnée de communications scientifiques sur l'occultisme, 1.1903, Hefte 17-19), afin que ce dernier puisse les lire ; ils se trouvent également aujourd'hui encore avec les œuvres imprimées de George. Et il demanda à Lechter de les lui renvoyer. Dans ces revues il n'y a pas cette fois de traduction de Dehmel, et malheureusement aucune annotation non plus de George. Mais elles nous renseignent sur le rapport de George avec ceux que l'on appelle les " Cosmiques ", et surtout sur ses expériences vécues dans le cercle d'Alfred Schuler, dans les années 1897 à 1903. Ce n'est pas le lieu ici d'évoquer les folles séances de ce groupuscule, les textes de Schuler et ses rituels magiques. Qu'ils aient éprouvé George et de quelle façon, un poème intitulé A.S., que l'on peut trouver dans la deuxième édition du recueil Jahr der Seele (L'année de l'âme), nous le fait déjà comprendre :

\section{A.S.}

« Elle a donc véritablement existé cette ronde ?

Des flambeaux/

Ont illuminé les visages blêmes ? Les vapeurs se sont élevées des calices/

Et ont enveloppé l'enfant-dieu ? Et tes paroles|

Nous ont tout rougissants emportés dans les mondes de la folie ?|

Nous perdons presque conscience; comme empoisonnés|

Par un lourd repas de fête, tout le jour nous chavirons.|

Nous les sentons toujours brûler les roses sur notre front. Souffrant/

Sous les regards curieux, dans l'éclat d'un ciel voilé ".
A.S.

So war sie wirklich diese runde? da fackeln Die bleichen angesichter hellten · dämpfe stiegen Aus schalen um den götterknaben und mit deinen worten

In wahneswelten grell-gerötet uns erhoben?

Dass wir der sinne kaum mehr mächtig • wie vergiftet Nach schlimmem prunkmahl taglang uns nicht fassten .

Stets um die stirn noch rosen brennen fühlten . leidend

Für neugierblicke in die pracht verhängter himmel.)

Même si l'on ne doit pas lire le texte de George comme le compte rendu direct d'un moment précis, ce poème fait la synthèse de plusieurs expériences et il s'y concentre le vécu d'un monde qui croyait que les pratiques occultes constituaient le dernier moyen par lequel il était encore possible de sauver Nietzsche et de restaurer les cultes archaïques. Il me semble que pour comprendre la position de George le terme de " curiosité " (" neugier ") mais aussi l'expression de " mondes en folie" (" wahneswelten ") sont particulièrement importants. C'est la curiosité qui l'a poussé à participer à de telles soirées et à faire les frais de " souffrances " qui ne consistaient pas seulement en maux de tête. Les éclats antisémites de Klages contre le Juif Wolfskehl ont mis fin, si l'on résume la situation un peu rapidement, à cette manifestation carnavalesque, innocente en apparence seulement, dans le quartier munichois de Schwabing, que Franziska zu Reventlow a judicieusement rebaptisé "Wahnmoching " (" le quartier des folies ") $)^{13}$. George en avait longtemps auparavant reconnu les dangers et les avait pour lui-même conjurés, pas seulement dans son poème de l'année 1899. Il les avait aussi formulés dans cette mise en garde qu'il avait adressée en 1901 à son ami Karl Wolfskehl, bien plus fortement contaminé par cette exaltation : " Ne confonds pas le prêtre avec le fakir, le 'vates' avec le mage, les esprits avec les fantômes ", avait-il écrit sur un billet qu'il avait remis un 
jour à Wolfskehl, à Munich. Significativement, on en retrouve le contenu sous forme d'aphorisme dans la septième livraison des Feuillets pour l'art de 1904, sous le titre Urgrundschwärmer (Les rêveurs enthousiastes de l'Idée première) : " Ne confondez pas Dieu avec l'idole, l'esprit avec le fantôme, le visionnaire avec la sorcière ". Voilà une recommandation qui sonne comme une sentence prononcée par George à l'encontre des "Cosmiques " : ils pervertissent ce en quoi George a foi : Dieu, l'esprit, le visionnaire. Dans cette septième livraison de la revue précisément, on trouve cette dernière marque d'ironie à l'égard des prétentieux explorateurs de l'au-delà (d'où le titre de mon article) : " Êtres nus / vous vous défaites de votre enveloppe et la rejetez, et parce qu'alors vous êtes saisis de froid et d'une sensation d'inconnu, vous croyez plonger dans l'infini (de la chair de poule en guise de frisson achérontique) ". Si cet aphorisme à valeur générale peut aussi être interprété comme visant les réformateurs du mouvement « Monte Verità " ", il se rapporte pourtant à ce poème connu du contemporain de George, Detlev von Liliencron, qui parut en 1893 et qui porte le titre Acherontisches Frösteln (Frisson achérontique). En voici les quatre derniers vers :

« Un vide alors sème l'effroi dans les forêts,|

Au travers des ramures dénudées apparaît un fleuve,/ Il conduit, comme en rêve, jusqu'à mon rivage, la barque/

Qui m'emporte de l'autre côté, dans le froid et le silence ".

(Dann ängstet in den Wäldern eine Leere, Durch kahle Äste wird ein Fluss sich zeigen, Der schläfrig an mein Ufer treibt die Fähre, Die mich hinüberholt ins kalte Schweigen.)

Les mauvais poètes se font passer pour des " êtres nus ", que ce soit Dehmel ou Liliencron ; ils se conforment aux tendances du temps, se mettent au service des goûts et des modes, et trahissent ainsi ce qui pour George représente une valeur suprême : l'art et le " divin ", la poésie comme ultime médiation de l'un à l'autre.

Me voilà définitivement parvenue à cet objet scandaleux de la création poétique de George, avec lequel j'ai commencé ma démonstration, à l'annonciation poétique, formulée par George, de son dieu " Maximin ". Après la mort soudaine d'un lycéen avec lequel il s'était lié d'amitié, George a créé un nouveau mythe ; il traite de la vie et de la mort de Maximin, présentées dans le cycle poétique ainsi intitulé, cycle qu'il a placé en 1907 au centre de son recueil Der siebente Ring (Le septième anneau). Quoique le recueil contienne surtout des poèmes écrits avant la mort du jeune homme, une fois qu'il a été publié, c'est à partir de son noyau central que l'ensemble des textes a été compris et interprété. Conformément à la volonté de George, nous lisons les cycles de poèmes qui composent Le septième anneau (le cycle de poésies critiques Zeitgedichte, le cycle Gestalten, marqué par l'influence des "Cosmiques ", le cycle de poésies amoureuses intitulé Gezeiten) à l'ombre de ce mythe, celui de la naissance d'un dieu, que George

lui-même a résumé dans son premier poème du cycle Maximin :

« Pour l'un tu es un enfant ; pour l'autre un ami.|

Je vois en toi le dieu/

Que je reconnais en tremblant,|

L'objet de ma fervente piété $»^{15}$.

(Dem bist du kind $\cdot$ dem freund.

Ich seh in dir den Gott

Den schauernd ich erkannt

Dem meine andacht gilt.)

Ce dieu doit son existence à la poésie, il ne vit qu'en elle et que par elle ; la nécessité a fait de lui la créature d'un poète. Dans un temps de crise très profonde, un temps où s'est perdue toute forme de divin, et un 


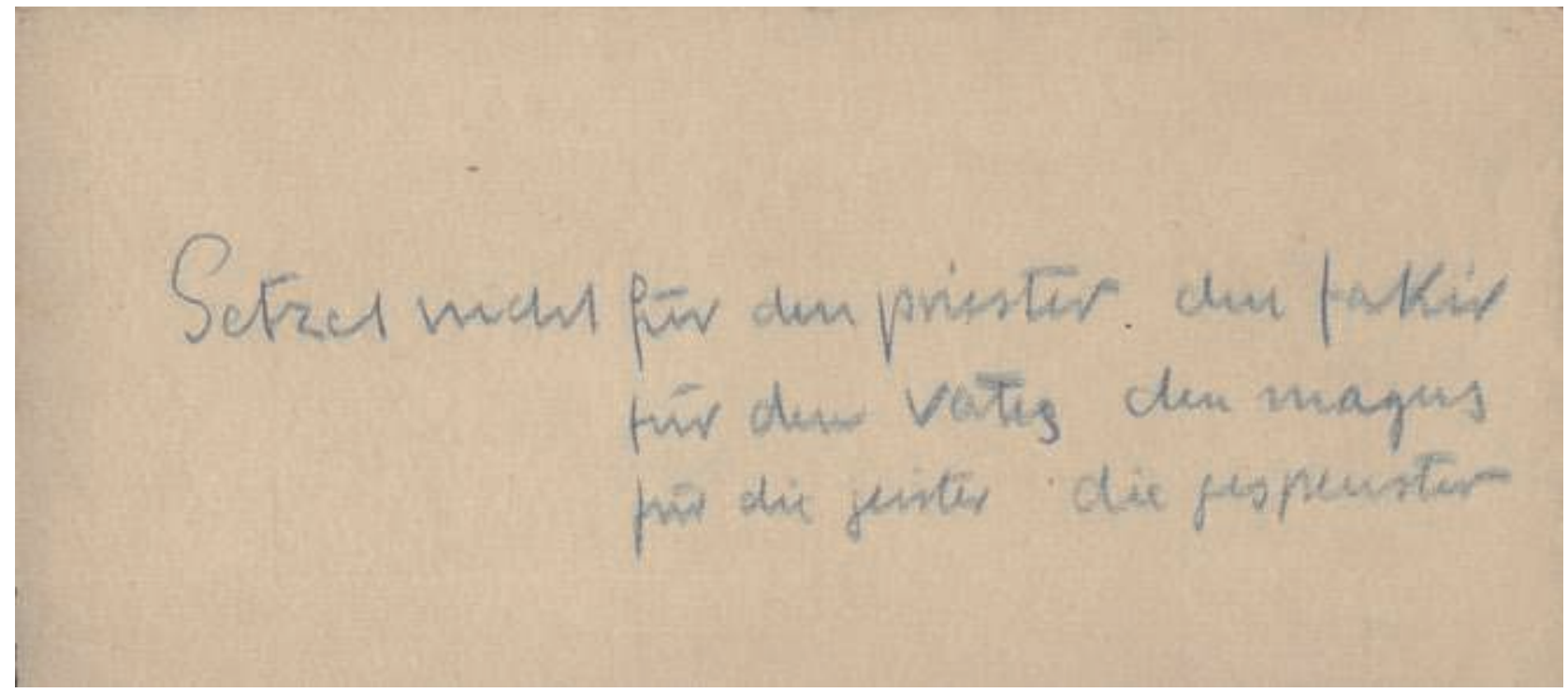

Mise en garde manuscrite de Stefan George à son ami Karl Wolfskehl (ca. 1903) contre la tentation des "prêtres ", " mages ", " fakirs " et autres " fantômes " (coll. WLB)

51 
temps où le doute s'est emparé de la propre mission du poète, le dieu " Maximin " sert à George d'autohabilitation. L'ange déjà participait d'un processus nécessaire de légitimation du poète, dans les temps de nécessité ; et comme " le messager ", le miroir, finalement a failli, le poète a conçu le dieu dont il s'est fait lui-même l'annonciateur et de son cercle d'amis il a fait une communauté de fidèles. De fait, on peut lire en 1910 dans les Feuillets pour l'art, sous le titre Das Göttliche (Le divin) le passage suivant : "Ce qui certifie le fonds d'humanité (il en va également ainsi pour toute forme naturelle), c'est le divin en l'humain : tout ce que l'homme peut produire ou créer vient de la bienveillance du divin ; la majeure partie de l'humanité, le troupeau, possède cela en elle de façon seconde, à un niveau enfoui, comme une étincelle... Les créateurs sont là pour attiser cette étincelle. Les créateurs possèdent le divin d'une façon première et immédiate : ce sont des archétypes, et se demander s'ils portent le divin en eux ou si c'est un dieu qui le leur a insufflé, est une question vaine - comme le fait de supposer un auteur pour chaque réalité ne fait que déplacer la frontière des causalités ». Telle est la profession de foi de George : les grands artistes et poètes sont des " archétypes ", des créateurs, des médiateurs. Gardiens du feu sacré, ils reconnaissent les étincelles dans les autres, le plus souvent « dans le feu des vrais regards $»^{16}$. Cette conception religieuse de l'art, le lecteur la rencontre souvent dans l'œuvre de George également associée à une exigeante critique culturelle, et il trouve cette poétique développée dans les nombreux textes en prose de la neuvième livraison des Feuillets pour l'art de l'année 1910. A cette époque, où l'on redécouvre les hymnes tardifs de Hölderlin, la poétique de George se renforce, en particulier sous l'influence de ce dernier. Mais Platon et le platonisme prennent également une signification plus profonde, les apports grecs et chrétiens sont reconnus comme les " formes essentielles premières " que féconde l'art poétique. George aspire à leur mariage sacré, tel qu'il avait été accompli par Goethe et Hölderlin ${ }^{17}$. Il s'inspire toujours plus de la tradition des arcanes, définit son royaume de l'esprit et le différencie radicalement de celui du savoir, s'empare de techniques poétiques qui permettent de crypter des messages, comme l'acrostiche ou d'autres combinaisons secrètes de lettres, et il définira plus tard son recueil de 1913/14 comme " un livre secret $\aleph^{18}$. L'on retrouve la contradiction entre l'espace fermé de l'arcane et l'espace ouvert du public ; le livre, qui s'adresse au petit cercle des amis proches, vise également un large lectorat. Ce recueil de L'étoile de l'alliance n'est pas resté une édition privée réservée à une élite mais, peu de temps avant que n'éclate la Première Guerre mondiale, il a été tiré à 2200 exemplaires, qu'il a fallu faire suivre d'un second tirage à 1 ooo exemplaires. Jusqu'en 1922, il n'y a pas moins de 15200 exemplaires de ce livre " secret " qui ont été publiés.

Il apparaît donc clairement que George a considéré que la constitution d'un petit cercle, d'un cénacle était une condition préalable à chaque renouvellement culturel et à chaque renouvellement artistique (comme il le formule déjà tôt dans les Feuillets pour l'art) ${ }^{19}$, qu'il utilise un vocabulaire, familier des contextes ésotérico-religieux, avec lequel il cultive un certain hermétisme (tout ce qui relève de l'occulte ou de l'audelà, le monde de la magie et des fantômes), mais qu'à partir de 1904 il prend largement ses distances avec cet univers.

Il est d'autant plus étonnant que dans les années 1909/1910 il ait été invité à l'abbaye de Neuburg, chez Alexander von Bernus, et que là-bas il ait également participé à des séances de spiritisme. Sur la proposition de George, une expérience fut même organisée : elle devait montrer un exemple, sur le corps à demi dénudé de Karl Wolfskehl, des effets d'une " concentration de rayons " émis par un bracelet ${ }^{20}$. La courte description de von Bernus fait comprendre au lecteur qu'il s'agit d'une farce préparée par George avec la complicité de Bernus, Wolfskehl et d'autres invités. Bernus assure également que la présence de George dans les séances de spiritisme avait un effet plus " paralysant que stimulant ". Nous avons connaissance de deux autres expériences venues du territoire " parasomatique ", mais qui ne nous sont rapportées que par le point de vue de Bernus et par ses commentaires. Dans les deux cas, il s'agit d'apparitions d'esprits. Dans l'un, Bernus croit qu'un esprit, peut-être celui du défunt lui-même, est venu annoncer sa mort, la nuit, à George. Une autre fois, il relate le combat désespéré de George contre un revenant dans une chambre d'hôte qui lui avait été réservée, une " cellule avec des voûtes gothiques ". George se serait vanté d'avoir résisté et de s'être montré d'une force supérieure, sans toutefois pouvoir chasser le revenant mais en réussissant à s'en accommoder. Là encore, on peut soupçonner George de n'avoir pas pris au sérieux cette croyance dans les esprits. Finalement la relation avec 
Bernus n'a duré que peu de temps. Quelques années plus tard seulement, George critiquait ouvertement les fréquentes visites de Melchior Lechter à l'abbaye ${ }^{21}$. Lechter lui-même était pourtant tout à fait conquis par " les nuits mystiques " qui se déroulaient là-bas, et il avait promis à un ami de lui raconter ce qu'il y avait abondamment vu " de beau, d'insolite et peutêtre d'occulte $"^{22}$.

Le dernier mot sur les revenants, les esprits et fantômes, sur les "Cosmiques " aussi bien que sur Bernus, revient à George, dans les dernières années de sa $v^{2}{ }^{23}$. Au cours d'une conversation, George tout surpris a confié que les "Cosmiques " " voulaient vraiment pratiquer la magie " mais qu'il leur en manquait les " ustensiles ". Il terminait en rapportant ce qu'il considérait comme " la remarque la plus profonde à propos de la croyance dans les fantômes ". La mère d'Alfred Schuler aurait dit à Wolfskehl : "Il m'est arrivé d'en voir un, mais d'y croire, ça, non !»

\section{Ute Oelmann}

(traduction Jean-Louis Elloy)

\footnotetext{
Notes

1 - Maximin. Un mémorial, édité par Stefan George, Berlin, 1907

2 - Robert Boehringer, Mon portrait de Stefan George, $2^{\mathrm{e}}$ éd. augmentée, Düsseldorf/München, 1968, p. 23 et suiv.

3 - Lettre d'Arthur Stahl à Stefan George, 17.7.1888, reproduite dans Boehringer, op. cit., p .23

4 - Stefan George, Franken (Les Francs), in Der siebente Ring, Berlin, 1907, p. 18 et suiv. Toutes les traductions de George, dans le texte comme dans les poèmes cités, sont de Jean-Louis Elloy.

5 - Voir Stefan George und der Symbolismus : une exposition de la Württembergische Landesbibliothek. Catalogue édité par Werner Paul Sohnle, Stuttgart, 1983

6 - L'appellation " les Cosmiques » ou " la ronde cosmique " désigne un cercle munichois très actif au tournant du $20^{\mathrm{e}}$ siècle. Formé essentiellement de Ludwig Klages (le théoricien), d'Alfred Schuler (" mystagogue " et " magicien ") et de Karl Wolfskehl, il s'attaque à la modernité, exalte un néo-paganisme très syncrétique et le célèbre en de pittoresques et carnavalesques cérémonies.

7 - Il s'agit des poèmes suivants extraits de Sagesse : I, 1 « Bon chevalier masqué... " et II, 4 " Mon Dieu m'a dit... "

8 - Ida Coblenz à George après le Noël de 1892, in Stefan George, Ida Coblenz. Correspondance, éditée par E. Höpker-Herberg, Stuttgart, 1983, p. 39

9 - Poème de Heinrich Hart, écrivain et critique proche des cercles naturalistes.

10 - George à Ida Coblenz, janvier à mars 1893, reproduit dans Stefan George, Ida Coblenz. Correspondance, p. 40
}

11 - Il s'agit du poème de George La visitation, qui a été plus tard intégré sans titre dans Le tapis de la vie, recueil de poèmes paru en 1900.

12 - Stefan George, Consécration, in Hymnen, Berlin, 189o, p. 2 et suiv.

13 - Cf. le roman-clé de Franziska zu Reventlow (figure saillante de la bohême de Schwabing, très ironique à l'égard des " Cosmiques ") Herrn Dames Aufzeichnungen, paru à Munich en 1913

14 - Mouvement fondé entre autres par Henri Oedenkoven et Ida Hoffmann à la fin du $19^{\mathrm{e}}$ siècle. Il connut un grand succès dans son refuge sur le Monte Verità, près du lac Majeur. Il réunissait des adeptes d'une vie naturelle, libérée des souillures du monde industriel, en quête de beauté. Il a séduit de grandes figures intellectuelles du temps, dont Hermann Hesse.

15 - Stefan George, Avent I, in Der siebente Ring, Berlin, 1907, p. 96

16 - Vers final du poème de George Neuen adel den ihr suchet (La nouvelle noblesse que vous recherchez) appartenant au recueil L'étoile de l'alliance (1914). Le poème se trouve p. 79.

17 - Cf. les textes de la neuvième livraison de 1910 : Für die Kunst, Das hellenische Wunder, Tote und lebendige Gegenwart, Diese und jene Welt, Das Göttliche, Der Heiland.

18 - Cf. les poèmes Dies ist reich des Geistes (Ceci est un royaume de l'esprit) et Ein wissen gleichfür alle heisst betrug (Un savoir égal pour tous est tromperie), extraits de L'étoile de l'alliance (p. 83 et 95), ainsi que l'Avantpropos à L'étoile de l'alliance dans l'édition complète de 1928 : " un livre qui aurait pu rester encore des années durant un livre secret ".

19 - Cf. Der Künstler und die Zeit, in Blätter für die Kunst, septième livraison, 1904 : « L'homme spirituel et artistique d'aujourd'hui doit forger ses valeurs en rupture complète avec l'opinion générale, avec toute exigence publique et quotidienne [...] Nous n'insistons pas pour le moment sur le fait que toute pensée créatrice, toute pensée libératrice provient des cercles occultes (des cénacles).

20 - Les informations et les citations proviennent de deux textes d'Alexander von Bernus, Sommertage und Sommernächte auf Stift Neuburg et Dichter und ihre Gesellen. Elles sont publiées dans le volume In Memoriam Alexander von Bernus. Ausgewählte Prosa aus seinem Werk, édité par Otto Heuschele, Heidelberg, 1966, p. 21-32 et 33-41.

21 - Ernst Morwitz à Karl Wolfskehl de la part de George, 15.10.1914, StGA

22 - Melchior Lechter à Friedrich Wolters, 16.7.1909, StGA

23 - Voir Clotilde Schlayer, Minusio. Chronik aus den letzten Lebensjahren Stefan Georges, éd. par Maik Bozza et Ute Oelmann, Göttingen, 2010, p. 40 